\title{
Kinetic modelling of blood glucose variation in a bioartificial pancreas
}

\author{
Tai-Horng Young ${ }^{\mathrm{a}, *}$, Jyh-Ping Hsu ${ }^{\mathrm{b}}$, Teng-Wang Nien ${ }^{\mathrm{b}}$ \\ ${ }^{a}$ Institute of Biomedical Engineering, College of Medicine and College of Engineering, National Taiwan University, Taipei 10016, Taiwan, ROC \\ ${ }^{\mathrm{b}}$ Department of Chemical Engineering, National Taiwan University, Taipei 10617, Taiwan, ROC
}

Received 29 October 2002; accepted 19 January 2003

\begin{abstract}
Methods are presented for assessing the performance of biomaterials for a bioartificial pancreas using a kinetic model of blood glucose variation. The model is composed of simultaneous differential equations that simulate variation of blood glucose concentration of rats outside the biomaterial and decrease of islet number inside the biomaterial. The resulting calculations agree well with the in vivo experimental data and indicate that the characteristics of bioartificial pancreas can be expressed as the actually working number of islets from the viewpoint of rats, regardless of the functioning of the bioartificial pancreas. The contributions of the capability of a biomaterial permeable to insulin and against immune rejection, and other factors that may induce cell death were involved in the actually working number of islets, i.e., the model used a lumped-parameter expression for assessment of the performance of biomaterials for a bioartificial pancreas. This model would be useful as a research tool for analysis of clinical investigation of bioartificial pancreas and physiological significance of blood glucose variation dynamics.
\end{abstract}

(C) 2003 Elsevier Science Ltd. All rights reserved.

Keywords: Bioartificial pancreas; Performance of biomaterials; Kinetic model

\section{Introduction}

The integration of biological cells or tissues into biomaterials in the development of artificial organs constitutes an important part of medical technology. The evaluation of the performance of biomaterials in biological systems is an important and complex matter in this situation. For example, the bioartificial pancreas [1-5] designed for controlling blood glucose levels in diabetes, involves the separation of transplanted islets from the host by a biomaterial's membrane. Besides preventing the rejection by the host immune system, the function of the biomaterial is to act as a permeable barrier membrane to small molecules such as nutrients, electrolytes, oxygen and secretory products. It is necessary to evaluate the ability of a biomaterial to support the islets secretion function and its ability to protect against immune rejections simultaneously. However, either the aggregation of islets inside the biomater-

*Corresponding author. Tel.: + 886-2-23123456x1455; fax: + 886-223940049.

E-mail address: thyoung@ha.mc.ntu.edu.tw (T.-H. Young). ial $[3,4,6,7]$, the encapsulation of the biomaterial by connective tissue, or the permeation of toxic cytokines $[8,9]$ may contribute to a decrease in the viability of islets, resulting in cell death by necrosis or apoptosis. Consequently, the evaluation of the performance of a biomaterial applied to the artificial pancreas must consider not only the fact that secreted insulin must effectively permeate through the biomaterial to gain access to the whole body, but also that the viability of islets surrounded by the biomaterial is a critical factor.

In this article, we show how a kinetic model of blood glucose variation may be employed to assess the viability and insulin-secreting function of transplanted islets enclosed within a biomaterial. The experimental data for nonfasting blood glucose levels of diabetic rats, diabetic rats transplanted with 200 free islets, and diabetic rats transplanted with bioartificial pancreas have been described previously [10] and are shown in Fig. 1. Our bioartificial pancreas was a 4-cm long poly (vinyl alcohol) (PVA) chamber, $2 \mathrm{~mm}$ in diameter, containing 80 pancreatic islets and two ends of the chamber were sealed with polytetrafluoroethylene plugs. Two types of PVA chambers, $\mathrm{m}-1$ and $\mathrm{m}-2$, were 


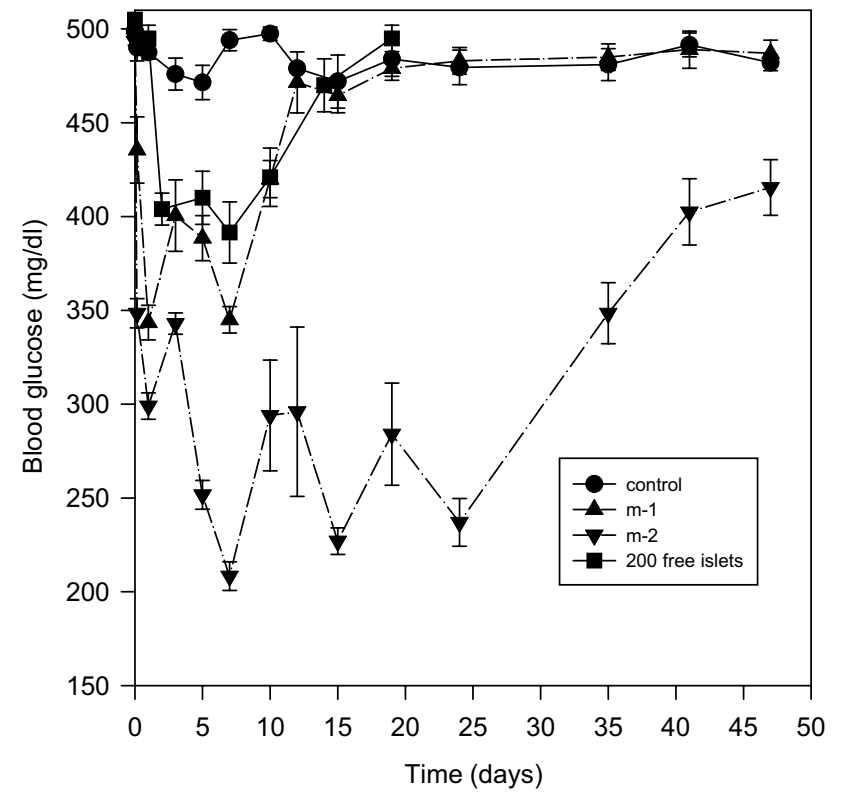

Fig. 1. Nonfasting blood glucose levels of diabetic rats, diabetic rats transplanted with 200 free islets, and diabetic rats transplanted with 80 islets seeded in the m-1 and m-2 tubular membrane chamber [10]. For each case, three diabetic rats underwent operation. Data are presented as the mean \pm standard deviation.

prepared in our laboratory. They were identical except for their outer surface wall (see Fig. 1 in Ref. [11]), but their permeability for insulin was significantly different $[11,12]$. As shown in Fig. 1, in the diabetic rats receiving m-1 chamber with 80 islets, nonfasting blood glucose levels dropped from $500 \pm 35 \mathrm{mg} / \mathrm{dl}$ to the lowest value $340 \pm 20 \mathrm{mg} / \mathrm{dl}$ only. In addition, the m-1 bioartificial pancreas only maintained insulin secretion activity for 10 days, which is similar to 200 free islets transplants showing slight decrease in nonfasting blood glucose levels for only 7 days. Conversely, nonfasting blood glucose levels in recipient diabetic rats were significantly decreased after the intraperitoneal transplantation of the m-2 chamber seeded with 80 islets compared to the diabetic rats without any peritoneal transplantation showing a hyperglycemic state. The lowest nonfasting blood glucose value obtained was about $210 \pm 22 \mathrm{mg} / \mathrm{dl}$ and nonfasting blood glucose levels less than $300 \mathrm{mg} / \mathrm{dl}$ were maintained for about 20 days. This indicates that the $\mathrm{m}-2$ bioartificial pancreas could provide improved permeability of insulin to normalize blood glucose levels and improved protection of islets from the immune system of the recipient.

\section{Theory}

In our previous theoretical analysis [10], the change in blood glucose levels of the diabetic rats after the intraperitoneal transplantation of bioartificial pancreas was divided into three stages. At the first stage, blood glucose concentration rapidly decreases because islets placed in the device responds to glucose stimulus. It is noted that the number of islets start to decrease at this stage. At the second stage, blood glucose concentration is kept at a constant low level. During this stage, the number of islets must be greater than a critical number, which is needed to maintain blood glucose concentration at a constant low level. At the final stage, blood glucose concentration rapidly rises to a hyperglycemic state because most of the transplanted islets are dead and the number of islets is lower than the critical number. However, the variation of blood glucose level of a rat is continuous, not broken during the period of transplantation, so the previous model is non-natural. In this study, a kinetic model describing continuous blood glucose variation by solving two simultaneous equations is proposed. Let $C$ be the blood glucose concentration of a rat, and $n_{\mathrm{i}}$ be the number of islets in the chamber. In Eq. (1), the blood glucose concentration variation with time, $t$, assuming to be uniformly distributed inside a rat, is a result of contributions from meal, $k_{1}$ that adds glucose and glucose metabolism that removes glucose.

$\frac{\mathrm{d} C}{\mathrm{~d} t}=k_{1}-k_{2} n_{\mathrm{i}}\left(C-C_{\mathrm{s}}\right)$,

where $k_{2}$ is a rate constant and $C_{\mathrm{s}}$ is a threshold of glucose concentration for islets secreting insulin according to the model described by Jaffrin et al. [13]. Here, we assume that glucose metabolism is proportional to the number of islets multiplied by $\left(C-C_{\mathrm{s}}\right)$ when blood glucose concentration exceeds $C_{\mathrm{s}}$. Note that Eq. (1) extends our previous model [10] to the case that the temporal variation of $C$ can be influenced by both $C$ itself and the meal fed to a rat.

It is noted that the number of islets, $n_{\mathrm{i}}$ is not a constant. Here, we assume that the variation of the number of islets can be described by two different mechanisms. This assumption can be reasoned by the result of aggregation of the islets, which has been reported by some investigators $[3,4,6,7]$. When the islets are packed in the chamber, the diffusion resistances for glucose and oxygen simply impair the viability of the central portion of the islet aggregate. Conversely, islets in the rim of the periphery of the clumps of necrotic islet cells can survive to a longer period. Let $N_{0}$ and $N_{\mathrm{c}}$ be the total number of islets initially seeded in the chamber and placed in the periphery of aggregation, respectively. Therefore, the temporal variations in the number of islets is described by

$n_{\mathrm{i}}=\left(N_{0}-N_{\mathrm{c}}\right) \exp \left(-k_{3} t\right)+N_{\mathrm{c}} \exp \left(-k_{4} t\right)$,

where $k_{3}$ and $k_{4}$ are rate constants, which are related to the mortality characteristics of islets in the transplanted chamber. Note that Eq. (2) extends our previous model [10] to take the nonuniformity of islets entrapped in a chamber into account. 


\section{Results}

The applicability of our model is justified by fitting it to the available experimental data. The governing equations were solved numerically based on a fourthorder Runge-Kutta algorithm, and the least squares criterion was adopted in the parameter estimation procedure. Fig. 2 shows that the present model can appropriately fit the variation of blood glucose level for transplantation of one m-1 chamber, one, two and three $\mathrm{m}-2$ chambers, and 200 free islets. The experimental data for implanting two and three $\mathrm{m}-2$ chambers also have been described previously [10], which could provide further better blood glucose normalization. The best fitted parameters $k_{1}, k_{2}, C_{\mathrm{s}}, k_{3}, k_{4}$ and $N_{\mathrm{c}}$ are summarized in Table 1. Regardless of the environment surrounding the transplanted islets, the values of $k_{1}, k_{2}$ and $C_{\mathrm{s}}$ are identical. It is reasonable that $k_{1}$ is constant because the meal of every rat can be considered similar. Likewise, $C_{\mathrm{s}}$ is constant for all the cases examined because the threshold of glucose concentration to secrete insulin for every rat is similar. In our model, $C_{\mathrm{s}}$ is $75 \mathrm{mg} /$ $\mathrm{dl}$, which is less than that assumed $(100 \mathrm{mg} / \mathrm{dl})$ by Reach and Jaffrin [14]. It is interesting to note that all rats have the same $k_{2}$. If $k_{2}$ is considered as a rate constant for the variation of blood glucose concentration in a rat, the same $k_{2}$ value for all rats can be reasoned by the fact that the rate constant is a function of temperature only. Therefore, the model considered in the present study is of typical kinetic nature. Eq. (1) implies that only one variable, the number of islets, determines the variation of blood glucose concentration. That is, the present model is a lumped-parameter one for evaluating the performance of bioartificial pancreas, which includes the interactions between seeded islets and biomaterial as well as the interactions between biomaterial and immune system of rat. Thus, it is a simple model to evaluate the performance of biomaterial in a biological system.

Eq. (2) shows that $k_{3}$ and $k_{4}$ are related to the rate of decrease of the number of islets; the smaller the $k_{3}$ and $k_{4}$ the lower the decrease rate of the number of islets, and the better the performance of a bioartificial pancreas. Table 1 shows that $k_{3}$ is greater than $k_{4}$ for all the cases examined, indicating the number of islets rapidly decreased initially, followed by a lower decrease rate. Since we assume that $N_{\mathrm{c}}$ is the number of islets following the lower decrease rate, the larger its value the more prolonged survival of islets. Table 1 also shows that the $k_{3}$ and $k_{4}$ of an m-2 chamber are smaller than those of an m-1 chamber. However, the $k_{3}$ values are larger for implanting two and three $\mathrm{m}-2$ chambers than those of an m-1 chamber. As will be discussed later, it is reasonable to attribute the greater $k_{3}$ values to more islets seeded in two or three $\mathrm{m}-2$ chambers. On the other hand, regardless of the number of the $\mathrm{m}-2$ chambers implanted in a rat, the $k_{4}$ values do not have difference among them. Conversely, the $k_{4}$ value for the case of an $\mathrm{m}-1$ chamber is significantly different with that for the case of an m-2 chamber. Combining the effect of $k_{4}$ with $N_{\mathrm{c}}$, the survival of islets is better for multiple-chamber implantation than single-chamber implantation, indicating the performance of one m-2 chamber can be improved by increasing the number of implanted chambers. Therefore, the performance of the bioartificial pancreas examined, from the best to the worst follows the order $\mathrm{m}-2$ (III) $>\mathrm{m}-2$ (II) $>\mathrm{m}-2$ (I) $>\mathrm{m}-1$; the letters in parentheses represent the number of implanted $\mathrm{m}-2$ chambers.

\section{Discussion}

Plotted in Fig. 3 is the variation of the number of islets for different bioartificial pancreases and 200 free islet transplants, calculated from Eq. (2) with the parameters shown in Table 1. For all the cases examined, the islet number rapidly decreases for the first 2 days, followed by a slow decrease rate. Therefore, $k_{3}$ is significantly larger than $k_{4}$, as shown in Table 1 . In addition, compared to the $N_{\mathrm{c}}$ values, Fig. 3 shows that the islet number has been less than $N_{\mathrm{c}}$ for the 1st or 2nd day. Hence, the first term on the right-hand side of Eq. (2) actually can be reduced to a linear form. Consequently, $k_{3}$ increases proportionally when the number of implanted islets (or $\mathrm{m}-2$ chambers) increased, as indicated in Table 1. As a result, it is reasonable that each m-2 chamber has similar $k_{3}$ value when multiplechamber implantation is used. However, multiplechamber implantation still can provide more total islet number, as shown in Fig. 3.

Although it is evident that the islet number decreases rapidly when the artificial pancreas is implanted into the peritoneal cavity of a rat, a question about the physiological meaning of rapid islet death remains to be interpreted. As described in the Introduction, many causes such as the aggregation of the islets inside the chamber $[3,4,6,7]$, the chamber encapsulated by connective tissue, or the permeation of cytokines $[8,9]$ may contribute to decrease viability of islet cells to result in cell death. However, it is uncertain whether these effects are able to result in islet death so rapidly. On the other hand, it has been suggested by Bergen et al. [15] that insulin secretion by rat islets placed in an artificial pancreas will be inhibited by the insulin existing inside the chamber. They recommended that this inhibition phenomenon should be considered in the design of a transplantation chamber. In fact, the diffusion rate of glucose and the insulin-secretion rate by islets are relatively rapid compared to the diffusion rate of insulin [12], so insulin concentration inside the chamber will accumulate to a high level to enhance this inhibition 

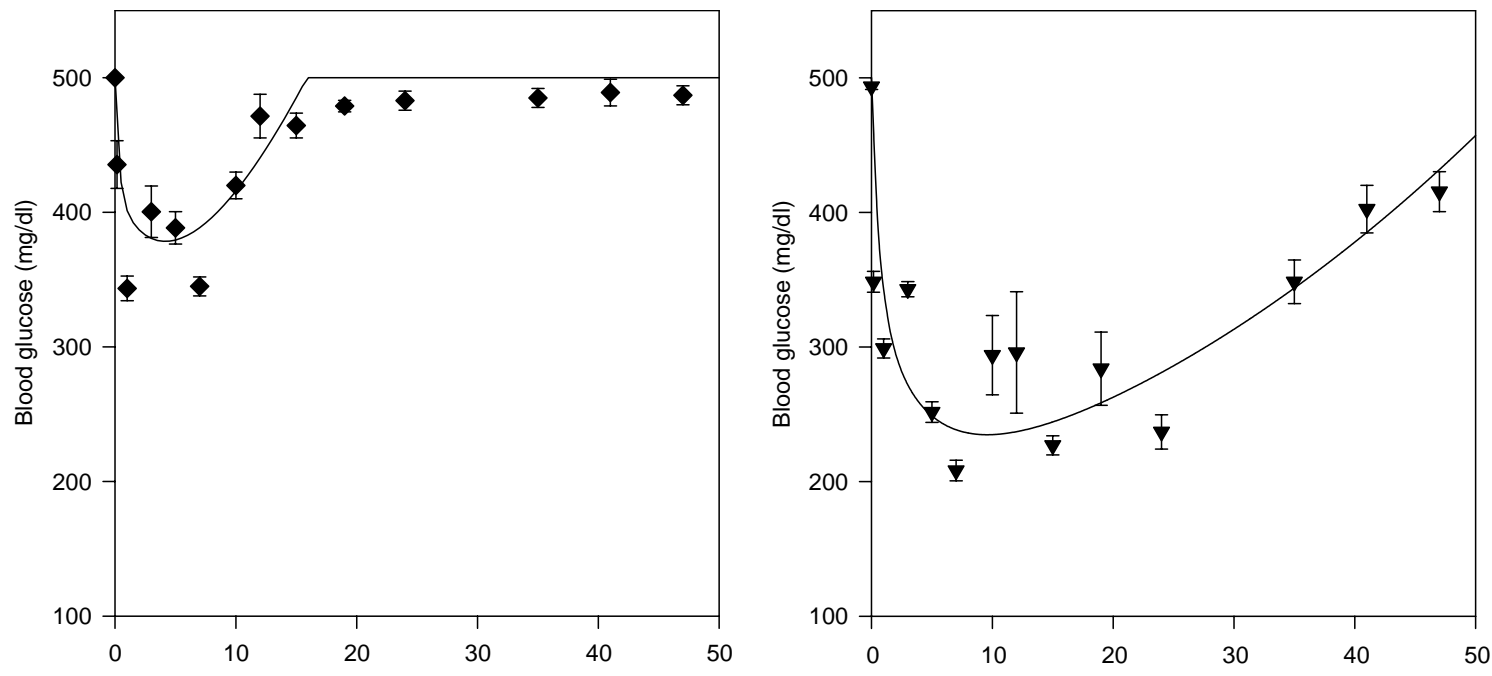

(a)

(b)

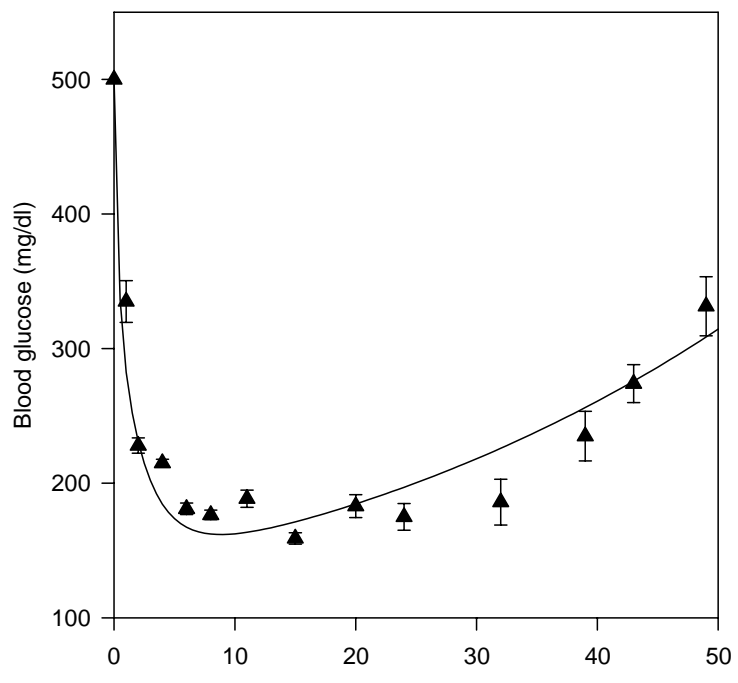

(c)

Time (days)

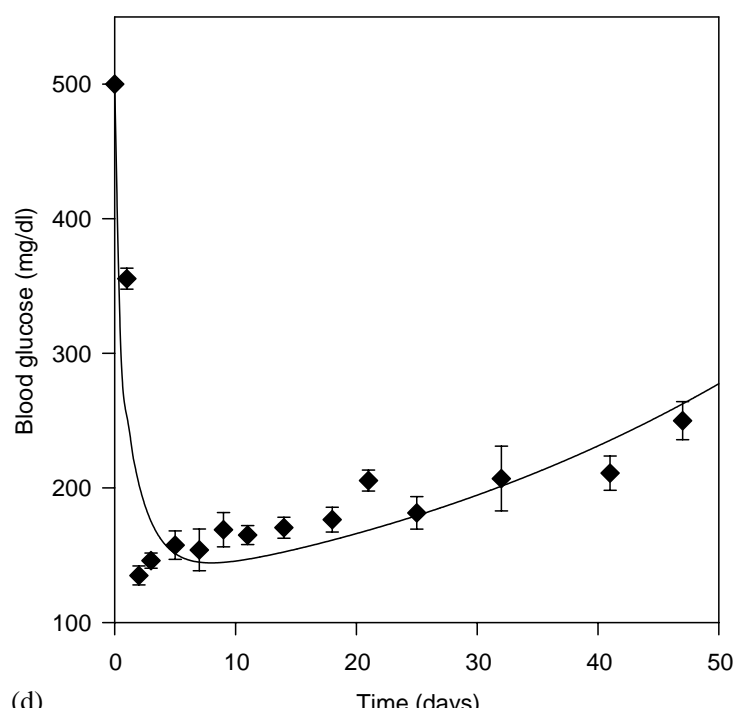

(d)

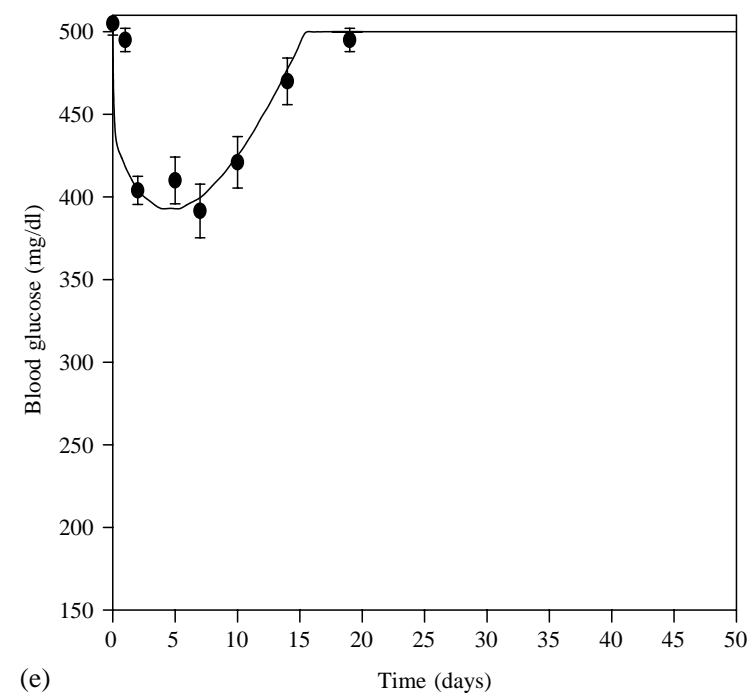

Fig. 2. The comparison in blood glucose levels between the experimental data [10] and the theoretical prediction. The parameters used in fitting the experimental data are shown in Table 1. (a) m-1; (b) m-2 (I); (c) m-2 (II); (d) m-2 (III); and (e) 200 free islets. (The letters in parentheses represent the number of implanted $\mathrm{m}-2$ chambers.) 
Table 1

Summary of the estimated parameters in fitting the experimental data shown in Fig. 2

\begin{tabular}{llllll}
\hline & 200 free islets & M-1 & M-2(I) & M-2(II) & M-2(III) $)^{\mathrm{a}}$ \\
\hline$k_{1}(\mathrm{mg} / \mathrm{dl} / \mathrm{day})$ & 30 & 30 & 30 & 30 & 30 \\
$k_{2}\left(\mathrm{day}^{-1}\right)$ & 0.01 & 0.01 & 0.01 & 0.01 & 75 \\
$C_{\mathrm{s}}(\mathrm{mg} / \mathrm{dl})$ & 75 & 75 & 75 & $2.8(=1.3 \times 2)$ & 75 \\
$k_{3}\left(\mathrm{day}^{-1}\right)$ & 12 & 3 & 1.3 & 0.03 & $4(=1.3 \times 3)$ \\
$k_{4}\left(\mathrm{day}^{-1}\right)$ & 0.1 & 0.1 & 0.03 & 45 & 0.03 \\
$N_{\mathrm{c}}$ & 15 & 15 & 25 & 55 \\
\hline
\end{tabular}

${ }^{\mathrm{a}}$ I, II, and III refer to the number of implanted $\mathrm{m}-2$ chambers.

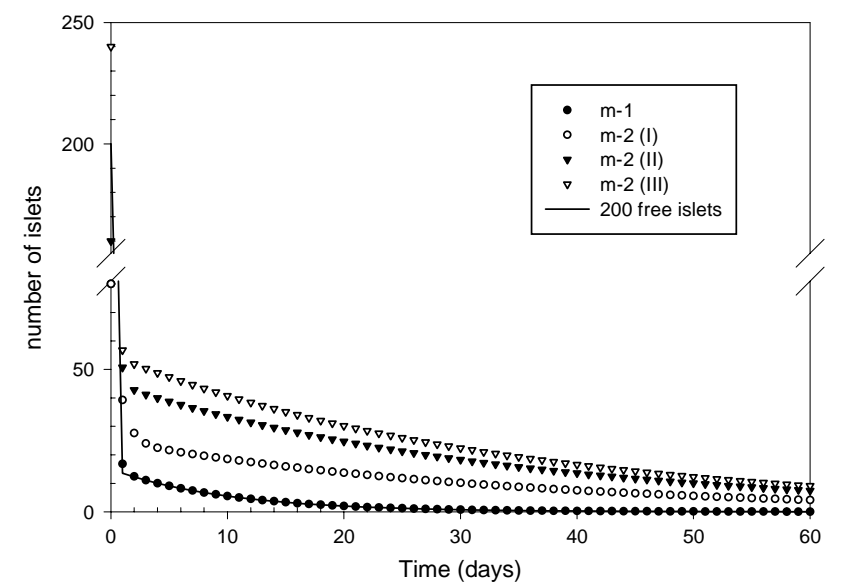

Fig. 3. The variation of the islet number for different bioartificial pancreases after transplantation, which was calculated from Eq. (2) based on the parameters of Table 1. (The letters in parentheses represent the number of implanted $\mathrm{m}-2$ chambers).

phenomenon. Therefore, the contributions of both cell death and inhibition of insulin secretion are involved in rapidly decreasing the number of islets in our model. In addition, due to low diffusion rate of insulin across the biomaterial, bioartificial pancreas cannot provide a sufficient amount of insulin even many islets secrete insulin inside the chamber to create a concentration gradient across the chamber. Taken together, from the viewpoint of rats, the number of working islets is far less than the actual number of islets placed in the chamber. Indeed, the presence of a biomaterial surrounding islets is to form a barrier for insulin permeation. That is, to say the present lumped-parameter model for evaluating the performance of bioartificial pancreas is to analyze the number of working islets from the viewpoint of rats. As shown in Fig. 3, transplantation of 200 free islets and m1 chamber almost have the same islet number variation, thus, similarly poor control of blood glucose concentration can be expected though their $k_{3}$ values are different to a certain degree. Regarding transplantation of 200 free islets, $k_{3}$ is $12 \mathrm{day}^{-1}$, indicating transplanted islets will be directly attacked by the host immune system.

Furthermore, $N_{\mathrm{c}}$ value can be considered as a simple index for the functioning of a bioartificial pancreas, and the smaller its value, the lower the performance of a biomaterial. The results of simulation show the $N_{\mathrm{c}}$ values are 15 and 25 for $\mathrm{m}-1$ and $\mathrm{m}-2$ chambers, respectively. This can be attributed to that the insulin concentration inside an m-1 chamber will accumulate to a higher value owing to its lower insulin diffusion rate [11,12], which is responsible for the smaller $N_{\mathrm{c}}$. In fact, transplantation of 200 free islets and m-1 chamber has the same $N_{\mathrm{c}}$ value.

Overall, the present model shows that the performance of a biomaterial applied in the artificial pancreas is determined by the $n_{\mathrm{i}}$ in the second term on the righthand side of Eq. (1), $k_{2} n_{\mathrm{i}}\left(C-C_{\mathrm{s}}\right)$, which reflects the effects of the insulin permeability across the biomaterial, the inhibition of insulin secretion, the microenvironment surrounding the islets, the chamber encapsulation, the immune rejection of transplanted islets, and others. However, for simplification, the present model did not take into account the islets placed in the device responding appropriately to glucose within a time frame that will lead to an overexcursion of blood glucose after a meal $[16,17]$. We have also ignored how to predict a sufficient amount of insulin to achieve reasonable diabetic control, especially when insulin secretion inhibition induced by high insulin concentration is considered. The present model focuses on the long-term blood glucose variation of a diabetic rat based on the actual number of working islets. The observation, from the viewpoint of rats, confirms only some seeded islets were functional despite 80 islets were seeded in the chamber. Therefore, the present study was performed to examine diabetic rats for the possibility of further reducing the blood glucose level by transplanting a sufficient number of islets. Based on Eq. (1) and Table 1, we can predict that a normal rat needs about 100 working islets to maintain its blood glucose level at $110 \mathrm{mg} / \mathrm{dl}$ at steady state. It is the goal of a bioartificial pancreas to support the maintenance of the number of working islets $(>100)$ in diabetic rats.

\section{Conclusion}

In this study, a kinetic model of blood glucose variation was used to assess the performance of 
bimaterials for a bioartificial pancreas. Although the model is composed of two differential equations and six parameters, the contributions of the capability of a biomaterial permeable to insulin and against immune rejection, and all factors that may induce cell death were involved in the actually working number of islets, i.e., the model is a lumped-parameter one for assessment of the performance of biomaterials for a bioartificial pancreas. Therefore, the higher the working number of islets the better the performance of a biomaterial. In addition, this model would be useful as a research tool for analysis of clinical investigation of bioartificial pancreas because it can give physiological significance such as the threshold of glucose concentration for islets secreting insulin and the number of working islets needed to maintain normal blood glucose level.

\section{Acknowledgements}

The authors thank the National Science Council of Taiwan, the Republic of China for their financial support of this research.

\section{References}

[1] Lim F, Sun AM. Microencapsulated islets as bioartificial endocrine pancreas. Science 1980;210:908-10.

[2] Altman JJ, Houlbert A, Callard P, McMillan P, Solomon BA, Rosen J, Galetti PM. Long term plasma glucose normalization in experimental diabetic rats with macroencapsulated implants of benign human insulinomas. Diabetes 1986;35:625-33.

[3] Sullivan SJ, Maki T, Borland KM, Mahoney MD, Solomon BA, Muller TE, Monaco AP, Chick WL. Biohybrid artificial pancreas: long-term implantation studies in diabetic, pancreatectomized dogs. Science 1991;252:718-21.

[4] Lacy PE, Hegre OD, Vazeou AG, Gentile FT, Dionne KE. Maintenance of normoglycemia in diabetic mice by subcutaneous xenograft of encapsulated islets. Science 1991;254:1782-4.
[5] Lanza RP, Beyer AM, Chick WL. Xenogenic humoral responses to islets transplanted in biohybrid diffusion chambers. Transplantation 1994;57:1371-5.

[6] Aung T, Kogire M, Inoue K, Fujisato T, Gu Y, Burczak K, Shinohara S, Mitsuo M, Maetani S, Ikada Y, Tobe T. Insulin release from a bioartificial pancreas using a mesh reinforced polyvinyl alcohol hydrogel tube. ASAIO J 1993;39:93-6.

[7] Aung T, Inoue K, Kogire M, Doi R, Kaji H, Tun T, Hayashi H, Echigo Y, Wada M, Imamura M, Fujisato T, Maetani S, Iwata H, Ikada Y. Comparison of various gels for immobilization of islets in bioartificial pancreas using a mesh-reinforced polyvinyl alcohol hydrogel tube. Transplant Proc 1995;27:619-21.

[8] Delaney CA, Pavlovic D, Hoorens A, Pipeleers DG, Eizirik DL. Cytkines induces deoxyribonucleic acid strand breaks and apoptosis in human pancreatic islet cells. Endocrinology 1997; 138:2610-4.

[9] Suarez-Pinzon WL, Strynadka K, Rabinovitch A. Destruction of rat pancreatic islet $\beta$-cells by cytokines involves the production of cytotoxic aldehydes. Endocrinology 1996;137:5290-6.

[10] Young TH, Chuang WY, Hsieh MY, Chen LW, Hsu JP. Assessment and modeling of poly (vinyl alcohol) bioartificial pancreas in vivo. Biomaterials 2002;23:3301-495.

[11] Young TH, Yao NK, Chuang WY, Chen LW. Use of a diffusion model for assessing the performance of poly (vinyl alcohol) bioartificial pancreases. J Biomed Mater Res 1998;40:385-91.

[12] Young TH, Yao NK, Chang RF, Chen LW. Evaluation of asymmetric poly (vinyl alcohol) membranes for use in the artificial islets. Biomaterials 1996;17:2131-7.

[13] Jaffrin MY, Reach G, Notelet D. Analysis of ultrafiltration and mass transfer in a bioartificial pancreas. J Biomech Eng 1988;110:1-10.

[14] Reach G, Jaffrin MY. Kinetic modeling as a tool for the deign of a vascular bioartificial pancreas: feedback between modeling and experimental validation. Comput Meth Programs Biomed 1990;32:277-85.

[15] Bergen JF, Mason NS, Scharp DW, Sparks RE. Insulin inhibition of islets in transplantation chambers. Artif Organs 1978;172: $144-50$.

[16] Sorensen J, Colton CK, Hillman RS, Soeldner JS. Use of a physiologic pharmacokinetic model of glucose homeostasis for assessment of performance requirements for improved insulin therapy. Diabetes Care 1982;5:148-56.

[17] Kraegen EW, Chisholm DJ, MacNamara ME. Timing of insulin delivery with meals. Horm Metab Res 1981;13:365-7. 